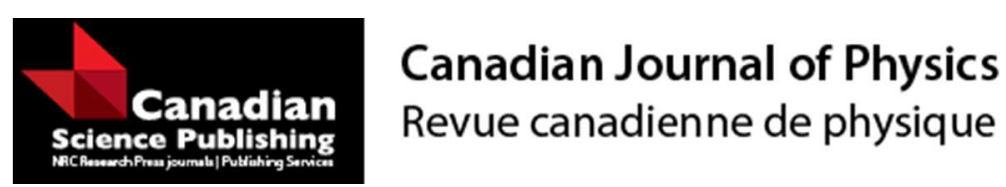

\title{
Practical Quantum Teleportation of an Unknown Quantum State
}

\begin{tabular}{|r|l|}
\hline Journal: & Canadian Journal of Physics \\
\hline Manuscript ID & cjp-2016-0758.R1 \\
\hline Manuscript Type: & Article \\
\hline Date Submitted by the Author: & 16 -Dec-2016 \\
\hline Complete List of Authors: & $\begin{array}{l}\text { Amin, Syed; National University of Sciences and Technology, School of } \\
\text { Natural Sciences } \\
\text { Khalique, Aeysha; National University of Sciences and Technology, School } \\
\text { of Natural Sciences }\end{array}$ \\
\hline Keyword: & $\begin{array}{l}\text { Quantum teleportation, Quantum information, Quantum communication, } \\
\text { Quantum Physics, Quantum entanglement }\end{array}$ \\
\hline
\end{tabular}

SCHOLARONE ${ }^{\text {th }}$

Manuscripts 


\title{
Practical Quantum Teleportation of an Unknown Quantum State
}

\author{
Syed Tahir Amin and Aeysha Khalique \\ School of Natural Sciences, National University of Sciences and Technology, H-12 Islamabad, Pakistan
}

(Dated: January 26, 2017)

\begin{abstract}
We present our model to teleport an unknown quantum state using entanglement between two distant parties. Our model takes into account experimental limitations due to contribution of multi-photon pair production of parametric down conversion source, inefficiency and dark counts of detectors and channel losses. We use a linear optics setup for quantum teleportation of an unknown quantum state by performing Bell state measurement by the sender. Our theory successfully provides a model for experimentalists to optimize the fidelity by adjusting the experimental parameters. We apply our model to a recent experiment on quantum teleportation and the results obtained by our model are in good agreement with the experiment results.
\end{abstract}

PACS numbers: 03.67.-a, 03.67.Hk, 03.67.Bg

\section{INTRODUCTION}

Quantum teleportation (QT) is the process of sending quantum state of any physical quantum system from one place to another without sending the system itself. The idea of QT, presented in 1992 [1] consists of disassembling of an unknown quantum state into purely classical information and quantum correlations and then later reconstructing the same quantum state by using this information.

Quantum teleportation is a key tool in quantum computation [2]. Theoretical proposals of quantum teleportation involve various variations like teleportation of atomic states between cavities [3, 4]. Many successful experimental attempts have been made to demonstrate QT, after the first demonstration in 1997 using entangled photons [5]. Laboratory demonstrations involve open destination QT [6], an entanglement swapping demonstration [7], two-bit composite system QT [8] and a fully integrated implementation of QT in which all key parts of the circuit are performed on a reconfigurable photonic chip [9]. In addition QT through a fiber link has been realized $[10,11]$, which, however is limited to $1 \mathrm{~km}$. Recently, QT over a distance of $16 \mathrm{~km}$ is demonstrated via free space links [12] using single entangled photons pair and over a distance of $100 \mathrm{~km}$ using parametric down conversion sources. Although QT demonstrated by above techniques transmits a state, having an overlap with the original state above the classical limit, but still these QT techniques are unable to proceed with $100 \%$ overlap. It is thus important to see the effect of various experimental parameters limiting the QT process.

Quantum teleportation has been analyzed for parametric down conversion (PDC) sources for entangled photon pairs but with approximate sources [13]. The effect of PDC sources on quantum communication protocols has recently being studied with threshold detectors [14]. In this paper we thoroughly analyze the multipair effect of the PDC sources including detector dark counts and inefficiencies with a model applicable to both photon number resolving and threshold detectors. The modeling with photon number resolving detectors can be used to circumvent the multipair effect and hence increase the fidelity, by post-selecting only single photon counts on the detectors. In this paper, we have also considered the limiting factors in long distance QT. Our theory relies on the model of resources presented in [15], where Bayesian approach is used to relate the counts on ideal detectors with unit efficiency and zero dark counts to inefficient detectors with dark counts.

Our results show that multipair production is counterproductive above certain value, though a high fidelity can be achieved for any value of detector inefficiency for very low dark counts and low pair production rate. This pair production rate is much lower than that used in the experiments since much higher pair production rates are practically used to keep the runtime of the experiment reasonable. Thus a trade-off is needed between minimum requirement of detector efficiency and channel transmission and maximum tolerable dark counts of detectors and the experimental runtime. Our model is useful to optimize these various experimental parameters for maximizing the fidelity for QT to any distance. The analysis of QT with practical parameters with varying distances yields that the effective communication distance is limited by detector efficiencies and dark counts. For detectors with unit efficiency and zero dark counts the fidelity saturates to a sub unit value for arbitrary distances. Our model fits well to a recent experiment [16], which has achieved teleportation over a distance of $100 \mathrm{~km}$.

Our paper proceeds as follows: In Sec. II, we provide background on practical resources, which concerns about sources generating entanglement and detectors models. In Sec. III, we compute the teleported quantum state using threshold detectors and incorporating inefficient entangled sources by Bell state measurement. In Sec. IV, we apply our model to a recent experiment and show the agreement between the two. Finally, we summarize our results and conclude in Sec. V. 


\section{BACKGROUND: QT AND PRACTICAL RESOURCES}

Entanglement is a resource in quantum communication (QC). Communication protocols like quantum teleportation rely on entanglement for long distance distribution of an unknown quantum state. The entanglement generation sources, however produce multiple pairs which effect the distributed state. Other affecting factors are inefficiencies and dark counts of the detectors. In this section we prepare a background for our model of QT including the above mentioned resources imperfections. We give a brief introduction to quantum teleportation process in Sec. II A and review the model of the resources in Sec. II B.

\section{A. Technique of QT}

Quantum teleportation is sending of quantum state of any physical system from one place to another without sending the physical system itself. Sender, Alice, wants to send an unknown quantum state of a photon, $|\varphi\rangle=\alpha|0\rangle+\beta|1\rangle$, to receiver Bob, where both of them are spatially separated. It is not possible to know the state of the photon by direct measurement and quantum no-cloning prohibits making copies of the state. Transmission of the state relies on the idea given in 1992 [1], where Alice and Bob use an entangled pair together with the unknown state. The process of teleportation is depicted in Fig. 1. The entangled pair is any one of the

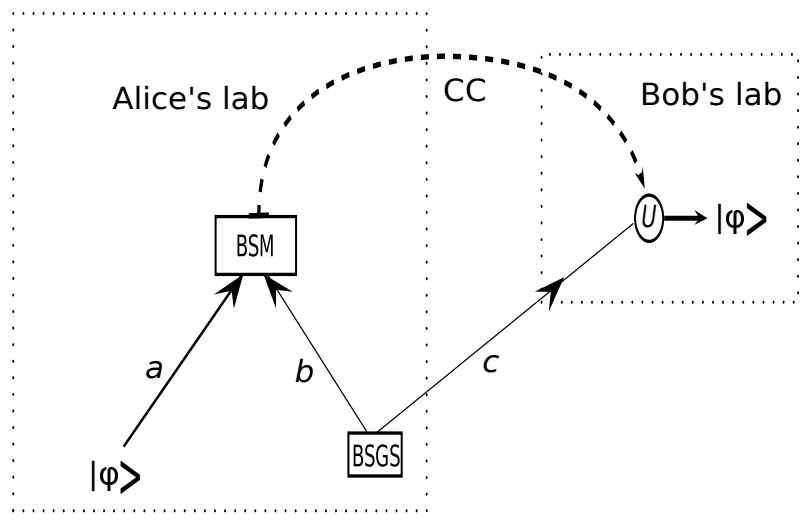

FIG. 1. Quantum teleportation: Bell State Generation Source (BSGS) produces an entangled pair in one of the Bell states in modes $b$ and $c$. Unknown state to be teleported, $|\varphi\rangle$ at mode $a$ is combined at Bell State Measurement (BSM). The result is communicated to Bob through classical communication (CC), who performs an appropriate unitary $U$ to get $|\varphi\rangle$

Bell states,

$$
\left|\phi^{ \pm}\right\rangle=\frac{1}{\sqrt{2}}(|00\rangle \pm|11\rangle) \quad\left|\psi^{ \pm}\right\rangle=\frac{1}{\sqrt{2}}(|01\rangle \pm|10\rangle),
$$

which are the maximally entangled states. Alice then performs a joint measurement, the Bell state measurement, on the unknown quantum system and on her part of the shared entangled pair, which collapses in one of the above mentioned Bell states. She then sends the result to Bob through classical communication. For the entangled pair in Bell state $\psi^{-}$, Bell state measurement resulting in $\psi^{-}$, projects Bob's part of the initially shared pair in the same state that Alice wanted to send. For Bell measurement resulting in $\phi^{-}, \phi^{+}$and $\psi^{+}$at Alice, Bob performs the unitaries $(U), \hat{\sigma}_{x}, \hat{\sigma}_{y}$ and $\hat{\sigma}_{z}$, respectively, on his particle, where

$$
\begin{aligned}
& \sigma_{x}=|0\rangle\langle 1|+| 1\rangle\langle 0|, \\
& \sigma_{y}=-i|0\rangle\langle 1|+i| 1\rangle\langle 0|, \\
& \sigma_{z}=-|1\rangle\langle 1|+| 0\rangle\langle 0|,
\end{aligned}
$$

are the Pauli operators, which transform Bob's particle in the same state as the desired unknown state. However, because of the imperfect resources, the teleportation is not perfect. We discuss these imperfect resources in the next section.

\section{B. Modeling of resources used in QT}

In this section, we give a review of a model of various resources used in QT, including associated imperfections.

Ideally single pair sources should be used as entangled pair sources, but they are not available, so spontaneous parametric down conversion (SPDC) sources are used. Here we give a model of a practical SPDC TYPEII source. Such a source emits polarization entangled photon pairs in some spatial modes, which are labeled as ' $b$ ' and ' $c$ ' . These photons have orthogonal polarizations labeled as horizontal (H) and vertical (V). Basically we want to find the state generated by SPDC TYPE-II, which is similar to the following Bell state,

$$
\begin{aligned}
\left|\psi^{-}\right\rangle_{\mathrm{bc}} & =\frac{1}{\sqrt{2}}(|\mathrm{HV}\rangle-|\mathrm{VH}\rangle)_{\mathrm{bc}}, \\
& =\frac{1}{\sqrt{2}}(|1001\rangle-|0110\rangle)_{b_{\mathrm{H}} b_{\mathrm{V}} c_{\mathrm{H}} c_{\mathrm{V}}} .
\end{aligned}
$$

The complete quantum state prepared by such an SPDC TYPE-II [17] is given as

$$
|\chi\rangle=\exp \left[i \chi\left(\hat{b}_{\mathrm{H}}^{\dagger} \hat{c}_{\mathrm{V}}^{\dagger}-\hat{b}_{\mathrm{V}}^{\dagger} \hat{c}_{\mathrm{H}}^{\dagger}+\hat{b}_{\mathrm{H}} \hat{c}_{\mathrm{V}}-\hat{b}_{\mathrm{V}} \hat{c}_{\mathrm{H}}\right)\right]|\mathrm{vac}\rangle,
$$

where, $\chi^{2}$ is proportional to the efficiency of generation of entangled photon pairs or photon pair production rate of the source. Normal ordered form of the above equation is [15],

$$
|\chi\rangle=\exp [2 \omega(\chi)] \exp \left[\phi(\chi)\left(\hat{b}_{\mathrm{H}}^{\dagger} \hat{c}_{\mathrm{V}}^{\dagger}-\hat{b}_{\mathrm{V}}^{\dagger} \hat{c}_{\mathrm{H}}^{\dagger}\right)\right]|\mathrm{vac}\rangle .
$$

Note that $|\mathrm{vac}\rangle=\left|0_{b_{H}} 0_{b_{V}} 0_{c_{H}} 0_{c_{V}}\right\rangle$. Ideally the quantum state of modes $b$ and $c$ corresponds to an entangled state 
of a pair of photons but in a realistic scenario it contains vacuum state and higher order Fock states as well.

Here we reprise the mathematical formalism of detecting $q$ photons such that $i$ photons are incident onto the detector [15]. For a photon number resolving detector with efficiency $\eta$ and dark count probability $\zeta_{\text {dc }}$, probability of such a detection is given as

$p_{\zeta_{\mathrm{dc}}, \eta}(q \mid i)=\frac{(1-\eta)\left(1-\zeta_{\mathrm{dc}}\right)}{1-\eta\left(1-\zeta_{\mathrm{dc}}\right)}\left(\frac{\eta}{1-\eta}\right)^{q}(1-\eta)^{i} G\left(i, q ; \eta, \zeta_{\mathrm{dc}}\right)$

for $i \geq q$ and

$p_{\zeta_{\mathrm{dc}}, \eta}(q \mid i)=\frac{(1-\eta)\left(1-\zeta_{\mathrm{dc}}\right.}{1-\eta\left(1-\zeta_{\mathrm{dc}}\right)}\left[\frac{1-\eta}{\eta} b\left(\eta, \zeta_{\mathrm{dc}}\right)\right.$
for $q>i$ where
\[ b\left(\eta, \zeta_{\mathrm{dc}}\right):=\left[1+\frac{1-\eta}{\eta \zeta_{\mathrm{dc}}}\right]^{-1} . \]

Also

$$
\begin{aligned}
G\left(\kappa, \lambda ; \eta, \zeta_{\mathrm{dc}}\right)= & \sum_{n=0}^{\infty}\left(\begin{array}{c}
\kappa \\
\lambda
\end{array}\right)\left(\begin{array}{c}
\kappa-\lambda+n \\
\kappa-\lambda
\end{array}\right)\left[b\left(\eta, \zeta_{\mathrm{dc}}\right)\right]^{n} \\
& \times\left[{ }_{2} F_{1}\left(-n,-\lambda ; \kappa-\lambda+1 ; \frac{\eta-1}{\eta}\right)\right]^{2}
\end{aligned}
$$

for $\kappa \geq \lambda$ and $G\left(\kappa, \lambda ; \eta, \zeta_{\mathrm{dc}}\right):=0$ for $\kappa<\lambda$. In the above equations, ${ }_{2} F_{1}$ is the hypergeometric function.

A threshold detector clicks if there are photons in a certain mode and does not click if there are no photons in that mode. Such a detector with efficiency $\eta$ and dark count probability $\zeta_{d c}$, does not click with probability

$$
\begin{aligned}
p_{\zeta_{\mathrm{dc}}, \eta}(\text { no click } \mid i) & =p_{\zeta_{\mathrm{dc}}, \eta}(q=0 \mid i) \\
& =\left(1-\zeta_{\mathrm{dc}}\right)\left\{1-\eta\left(1-\zeta_{\mathrm{dc}}\right)\right\}^{i} .
\end{aligned}
$$

The first term in Eq. (10) is the probability that the threshold detector does not click and that there is no dark count. The second term is the probability that $i$ photons are incident on the detector but the detector does not detect any incident photon. The probability of getting a click is then,

$$
\begin{aligned}
p_{\zeta_{\mathrm{dc}}, \eta}(\operatorname{click} \mid i) & =1-p_{\zeta_{\mathrm{dc}}, \eta}(\text { no click } \mid i) \\
& =1-\left(1-\zeta_{\mathrm{dc}}\right)\left\{1-\eta\left(1-\zeta_{\mathrm{dc}}\right)\right\}^{i}
\end{aligned}
$$

There are four detectors, one for each of the four spatial modes. Using threshold detectors, the posterior conditional probability for any read out $(i j k l)$, with four ideal detectors, with unit efficiency and zero dark counts, would have yielded [15]

$$
\begin{aligned}
P_{i j k l}^{q r s t} & =P(i j k l \mid q r s t) \\
& =\frac{p(q r s t \mid i j k l) p(i j k l)}{\sum_{i_{1} j_{1} k_{1} l_{1}}^{\infty} p\left(q r s t \mid i_{1} j_{1} k_{1} l_{1}\right) p\left(i_{1} j_{1} k_{1} l_{1}\right)} .
\end{aligned}
$$

Here, $p(i j k l)$ is the probability that ideally $\{i j k l\}$ photons are measured on the four detectors. Since the four detectors are independent of each other, we get,

$$
p(q r s t \mid i k j l)=p(q \mid i) p(r \mid j) p(s \mid k) p(t \mid l) .
$$

All transmission and other coupling losses are included in detector efficiency.

Equipped with the model of the resources, we present our model of quantum teleportation in the next section.

\section{MODELING OF PRACTICAL QT}

ditions of the resources discussed in the last section. Sec. III A we compute the pure teleported quantum state and its conditioned probability using ideal photon discriminating detectors in a Bell state measurement. In Sec. III B, we relate this state to that produced by practical threshold detectors in the form of a mixed density matrix. We then find the fidelity of the teleported state in Sec. III C.

\section{A. Postmeasurement teleported quantum state using ideal detectors}

Using the resources presented in the previous section we are now in a position to derive the teleported quantum state. We start with the quantum state produced by PDC-Type II source given in Eq. (5) and unknown quantum state in spatial mode $a$ as $|\varphi\rangle_{a}=\alpha|0\rangle+\beta|1\rangle$, which in $H-V$ modes takes the form $|\varphi\rangle_{a}=\alpha\left|1_{H} 0_{V}\right\rangle+\beta\left|0_{H} 1_{V}\right\rangle$. The composite state of photons in modes $a, b$ and $c$ will be

$$
\begin{aligned}
|\psi\rangle_{\mathrm{abc}}= & |\varphi\rangle_{\mathrm{a}} \otimes|\chi\rangle_{\mathrm{bc}} \\
= & \alpha \exp [2 \omega(\chi)] \exp \left[\phi(\chi)\left(\hat{b}_{\mathrm{H}}^{\dagger} \hat{c}_{\mathrm{V}}^{\dagger}\right)\right] \\
& \times \exp \left[-\phi(\chi)\left(\hat{b}_{\mathrm{V}}^{\dagger} \hat{c}_{\mathrm{H}}^{\dagger}\right)\right] \hat{a}_{\mathrm{H}}^{\dagger}|\mathrm{vac}\rangle_{\mathrm{abc}} \\
& +\beta \exp [2 \omega(\chi)] \exp \left[\phi(\chi)\left(\hat{b}_{\mathrm{H}}^{\dagger} \hat{c}_{\mathrm{V}}^{\dagger}\right)\right] \\
& \times \exp \left[-\phi(\chi)\left(\hat{b}_{\mathrm{V}}^{\dagger} \hat{c}_{\mathrm{H}}^{\dagger}\right)\right] \hat{a}_{\mathrm{V}}^{\dagger}|\mathrm{vac}\rangle_{\mathrm{abc}} .
\end{aligned}
$$

Bell state measurement is then performed by combining modes $a$ and $b$ on a balanced beam splitter. The four tuple of detectors then measure the photons in the two polarization modes in each of $a$ and $b$. First we consider that the four detectors used in the Bell state measurement were perfect, having unit efficiency $(\eta=1)$ and no detector dark counts $\left(\zeta_{d c}=0\right)$. Applying balanced beam splitter transformation $U_{\mathrm{BS}}$ to modes $a$ and $b$ using the rule [15]

$$
\begin{aligned}
& \hat{a}_{\mathrm{H}}^{\dagger} \stackrel{U_{\mathrm{BS}}}{\longrightarrow} \frac{1}{\sqrt{2}}\left(\hat{a}_{\mathrm{H}}^{\dagger}-\hat{b}_{\mathrm{H}}^{\dagger}\right), \quad \hat{b}_{\mathrm{H}}^{\dagger} \stackrel{U_{\mathrm{BS}}}{\longrightarrow} \frac{1}{\sqrt{2}}\left(\hat{a}_{\mathrm{H}}^{\dagger}+\hat{b}_{\mathrm{H}}^{\dagger}\right), \\
& \hat{a}_{\mathrm{V}}^{\dagger} \stackrel{U_{\mathrm{BS}}}{\longrightarrow} \frac{1}{\sqrt{2}}\left(\hat{a}_{\mathrm{V}}^{\dagger}-\hat{b}_{\mathrm{V}}^{\dagger}\right), \quad \hat{b}_{\mathrm{V}}^{\dagger} \stackrel{U_{\mathrm{BS}}}{\longrightarrow} \frac{1}{\sqrt{2}}\left(\hat{a}_{\mathrm{V}}^{\dagger}+\hat{b}_{\mathrm{V}}^{\dagger}\right) .
\end{aligned}
$$


The resultant three spatial modes quantum state, $U_{\mathrm{BS}}|\psi\rangle_{\mathrm{abc}}$, after passing through balanced beam splitter is projected onto subspace using the projection operator

$$
\begin{aligned}
\Pi_{a_{\mathrm{H}} a_{\mathrm{V}} b_{\mathrm{H}} b_{\mathrm{V}}}^{(i k j l)}:= & (|i\rangle\langle i|)_{a_{\mathrm{H}}} \otimes(|j\rangle\langle j|)_{a_{\mathrm{V}}} \otimes(|k\rangle\langle k|)_{b_{\mathrm{H}}} \\
& \otimes(|l\rangle\langle l|)_{b_{\mathrm{V}}} \otimes I_{c_{\mathrm{H}}} \otimes I_{c_{\mathrm{V}}} .
\end{aligned}
$$

Here $(|n\rangle\langle n|)_{d_{\mathrm{H}}}$, represents projection operator on some Fock state $|n\rangle$ in mode $d$ having horizontally polarized photon and analogously for vertically polarized photon. The normalized quantum state obtained after apply- ing the projection operator (16) is the postmeasurement state given by

$$
\frac{\Pi_{a_{\mathrm{H}} a_{\mathrm{V}} b_{\mathrm{H}} b_{\mathrm{V}}}^{i j k l}\left(\boldsymbol{U}_{\mathrm{BS}}^{\mathrm{ab}}|\psi\rangle_{\mathrm{abc}}\right)}{\sqrt{p(i j k l)}}=|i j k l\rangle_{a_{\mathrm{H}} a_{\mathrm{V}} b_{\mathrm{H}} b_{\mathrm{V}}} \otimes|\Phi\rangle_{c_{\mathrm{H}}, c_{\mathrm{V}}}^{i j k l},
$$

with the first factor being the Fock state with modes $a_{\mathrm{H}}, a_{\mathrm{V}}, b_{\mathrm{H}}, b_{\mathrm{V}}$ having $i, j, k, l$ photons respectively, and

$$
\begin{aligned}
& |\Phi\rangle_{c_{\mathrm{H}}, c_{\mathrm{V}}}^{i j k l}=\frac{1}{\left[\alpha^{2}(i+k-1) !(j+l) !(i-k)^{2}+\beta^{2}(i+k) !(j+l-1) !(l-j)^{2}\right]^{\frac{1}{2}}} \\
& \quad \times[\alpha \sqrt{(i+k-1) !(j+l) !}(i-k)|j+l, i+k-1\rangle+\beta \sqrt{(i+k) !(j+l-1) !}(l-j)|j+l-1, i+k\rangle]_{c_{\mathrm{H}}, c_{\mathrm{V}}}
\end{aligned}
$$

is the state on spatial mode $c$ such that $\{i j k l\}$ photons are detected on the other two modes. Equation(18) thus gives the teleported state. The corresponding probability of the hypothetical ideal measurement readout $(i j k l)$

$$
\begin{aligned}
p(i j k l) & =\frac{[\tanh \chi]^{2(i+j+k+l-1)}}{\cosh ^{4} \chi\left(2^{i+j+k+l} i ! j ! k ! l !\right)} \\
& \times\left[\alpha^{2}(i+k-1) !(j+l) !(i-k)^{2}\right. \\
& \left.+\beta^{2}(i+k) !(j+l-1) !(l-j)^{2}\right] .
\end{aligned}
$$

is the probability that pure quantum state $|\Phi\rangle_{c_{\mathrm{H}}, c_{\mathrm{V}}}^{i j k l}$ will be detected using ideal detectors in the Bell state measurement.

\section{B. Postmeasurement teleported quantum state using threshold detectors}

We find the teleported quantum state in previous section, using ideal detectors having efficiency $\eta=1$ and dark counts probability $\zeta_{\mathrm{dc}}=0$. Since in practical scenario the detectors are not perfect but have efficiency, $\eta<1$, and non-zero dark counts, $\zeta_{\mathrm{dc}} \neq 0$, we can neither find the post-measured pure resultant quantum state nor the probability of its occurrence of the remaining mode $c$. However for any read out $(i j k l)$ of four tuple detectors we can calculate its posterior probability $P_{i j k l}^{q r s t}=p(i j k l \mid q r s t)$ using Bayes theorem (12). We can achieve our task using the conditional probabilities $p(q r s t \mid i j k l)$ in Eq. (13). Hence, the resultant teleported quantum state of the remaining mode $c$, after the Bell state measurement using threshold detectors yielding ac- tual read out (qrst), is a mixed state of the form

$$
\varrho_{c_{\mathrm{H}}, c_{\mathrm{V}}}^{q r s t}=\sum_{i j k l} P_{i j k l}^{q r s t}|\Phi\rangle_{c_{\mathrm{H}}, c_{\mathrm{V}}}^{i j k l}\langle\Phi|
$$

Using Eqs. (19) and (12), we calculate

$$
\begin{aligned}
P_{i j k l}^{q r s t}= & \frac{p(q r s t \mid i j k l)[\tanh \chi]^{2(i+j+k+l)}}{Z^{q r s t} \times\left(2^{i+j+k+l} i ! j ! k ! l !\right)} \\
& \times\left[\alpha^{2}(i+k-1) !(j+l) !(i-k)^{2}\right. \\
& \left.+\beta^{2}(i+k) !(j+l-1) !(l-j)^{2}\right],
\end{aligned}
$$

with

$$
\begin{gathered}
Z^{q r s t}=\sum_{i_{1} j_{1} k_{1} l_{1}}^{\infty} \frac{p\left(q r s t \mid i_{1} j_{1} k_{1} l_{1}\right)[\tanh \chi]^{2\left(i_{1}+j_{1}+k_{1}+l_{1}\right)}}{2^{i_{1}+j_{1}+k_{1}+l_{1}} i_{1} ! j_{1} ! k_{1} ! l_{1} !} \\
\times E^{\left(i_{1} j_{1} k_{1} l_{1}\right)}
\end{gathered}
$$

where,

$$
\begin{aligned}
E^{\left(i_{1} j_{1} k_{1} l_{1}\right)}= & {\left[\alpha^{2}\left(i_{1}+k_{1}-1\right) !\left(j_{1}+l_{1}\right) !\left(i_{1}-k_{1}\right)^{2}\right.} \\
& \left.+\beta^{2}\left(i_{1}+k_{1}\right) !\left(j_{1}+l_{1}-1\right) !\left(l_{1}-j_{1}\right)^{2}\right] .
\end{aligned}
$$

Thus we have developed a closed form of the teleported state at Bob's end incorporating faulty detectors and multipair SPDC sources. This state is not exact replica of the desired state to be sent due to the imperfections. We find the overlap between the two states in the next section.

\section{Fidelity of final state at receiver}

In order to find the overlap of the quantum state obtained by Bob with the actual quantum state $|\varphi\rangle=$ 
$\alpha|10\rangle+\beta|01\rangle$, that Alice wanted to teleport, we use fidelity $(F)$ as a measure, with $0 \leq F \leq 1$. For $F=0$, there is no overlap, whereas for $\bar{F}=\overline{1}$, the overlap is maximum. Fidelity

$$
F_{c_{\mathrm{H}}, c_{\mathrm{V}}}^{q r s t}=\sqrt{\left\langle\varphi\left|\varrho_{c_{\mathrm{H}}, c_{\mathrm{V}}}^{q r s t}\right| \varphi\right\rangle}
$$

is calculated using Eqs. (18) and (20) as

$$
\begin{aligned}
F_{c_{\mathrm{H}}, c_{\mathrm{V}}}^{q r s t} & =\left[\beta^{2}\left(P_{2000}^{q r s t}+P_{0020}^{q r s t}\right)+\alpha^{2}\left(P_{0200}^{q r s t}+P_{0002}^{q r s t}\right)\right. \\
& \left.+P_{1100}^{q r s t}+P_{0011}^{q r s t}+\left(\alpha^{2}-\beta^{2}\right)^{2}\left(P_{1001}^{q r s t}+P_{0110}^{q r s t}\right)\right]^{\frac{1}{2}}
\end{aligned}
$$

Using Eq. (21) in above equation, finally we have the fidelity of the teleported state in the following form

$$
\begin{aligned}
F_{c_{\mathrm{H}}, c_{\mathrm{V}}}^{q r s t}= & {\left[\frac { \operatorname { t a n h } ^ { 4 } \chi } { 2 \times Z ^ { q r s t } } \left(\alpha^{2} \beta^{2}\{(p(\text { qrst } \mid 2000)+p(\text { qrst } \mid 0020)+p(q r s t \mid 0200)+p(\text { qrst } \mid 0002)\}\right.\right.} \\
& \left.\left.+\frac{1}{2}\left\{p(q r s t \mid 1100)+p(\text { qrst } \mid 0011)+\frac{1}{2}\left(\alpha^{2}-\beta^{2}\right)^{2}(p(q r s t \mid 1001)+p(q r s t \mid 0110))\right\}\right)\right]^{\frac{1}{2}}
\end{aligned}
$$

Note that $p(q r s t \mid i j k l)$ are the conditional probabilities of detecting the photons by four detectors given by Eq. (13) and value of $Z^{\text {qrst }}$ is given by Eq. (22). Thus we have developed a closed form solution of the fidelity between the teleported state and the actual desired state.

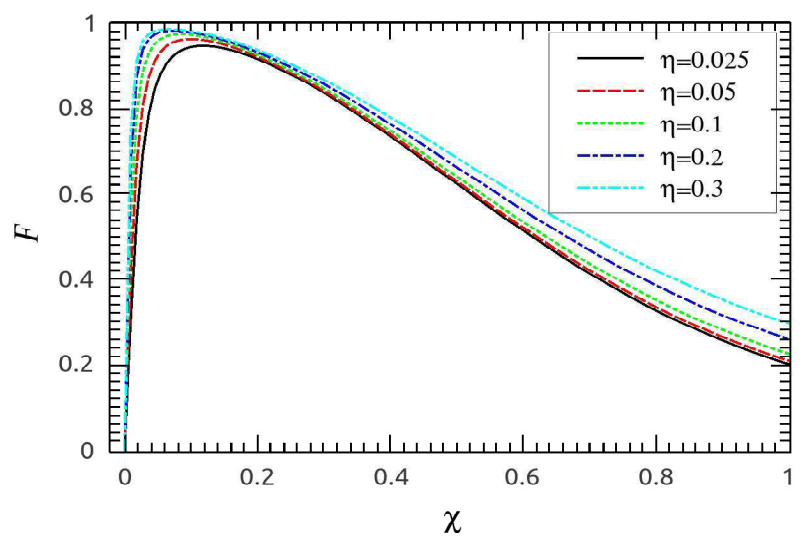

FIG. 2. (Color online) Plot of fidelity $F$ against the square root of photon-pair production rate, $\chi$ for dark-count probabilities, $\zeta_{\mathrm{dc}}=10^{-5}$, and various efficiencies $\eta=0.025$ to 0.3 from the curves of lowest to highest fidelity.

We now check the effect of the main limiting parameters in our QT theory for threshold detectors. We plot fidelity against square root of the photon pair production rate $\chi$, for fixed dark counts probability $\zeta_{\mathrm{dc}}=10^{-5}$ and different detectors efficiencies in Fig. 2. From Fig. 2 it

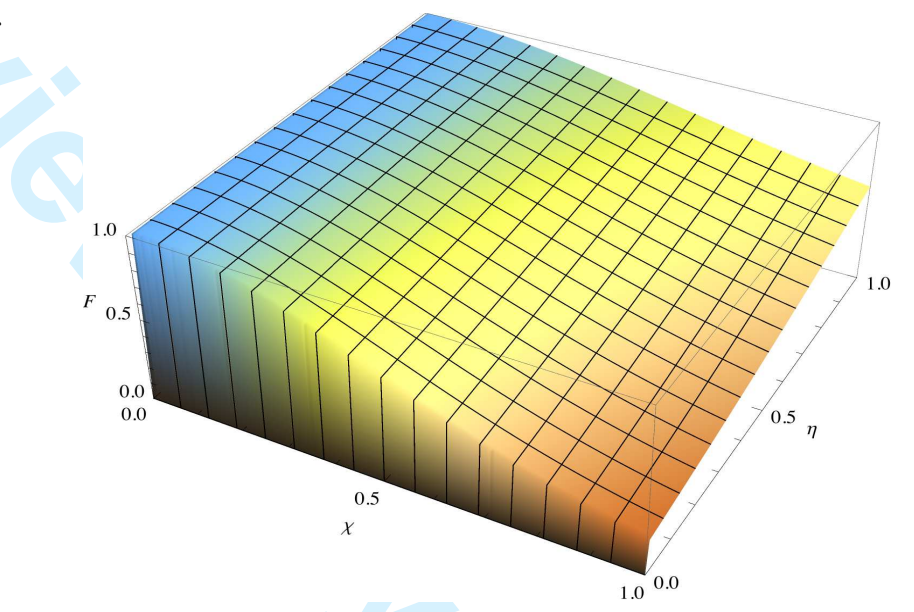

FIG. 3. (Color online) 3D plot showing variation of fidelity $F$ with both the pair production rate $\chi$ and the efficiency $\eta$ for dark-count probability, $\zeta_{\mathrm{dc}}=0$

is clear that above the classical limit, fidelity does not change prominently with changing detector efficiencies. It should be noted that there is region for some small value of $\chi$ where we can achieve unit fidelity for zero dark counts, irrespective of the detectors efficiencies, which is evident from 3D plot of fidelity with $\chi$ and $\eta$ in Fig 3 . Variation of the fidelity with $\chi$ for different dark count probabilities is given in Fig. 4, which shows that high dark counts effect the fidelity dominantly. Fig. 5 shows the variation of the fidelity vs distance. Fidelity saturates for certain distance but as soon as the dark counts 


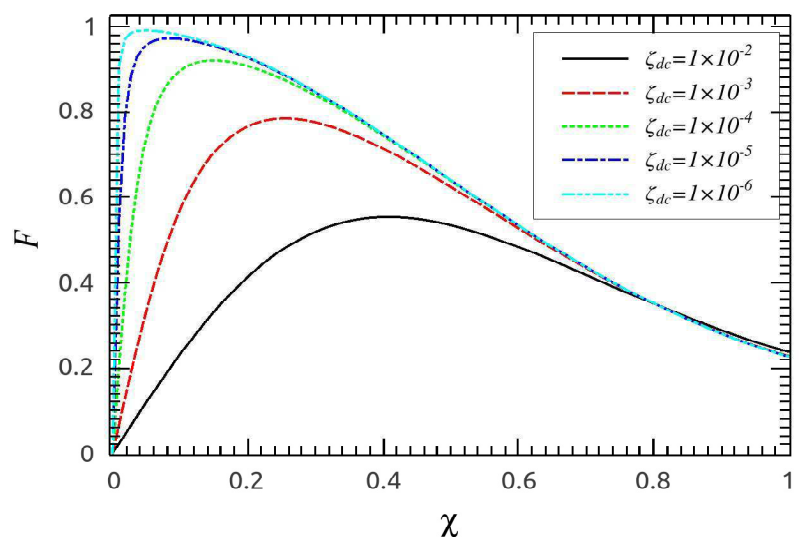

FIG. 4. (Color online) Plot of fidelity $F$ against the square root of photon-pair production rate, $\chi$ for fixed efficiency $\eta=$ 0.1 and dark-count probabilities, $\zeta_{\mathrm{dc}}=10^{-2}$ to $10^{-6}$ from the curves of lowest to highest fidelity.

become effective, it drops down suddenly.

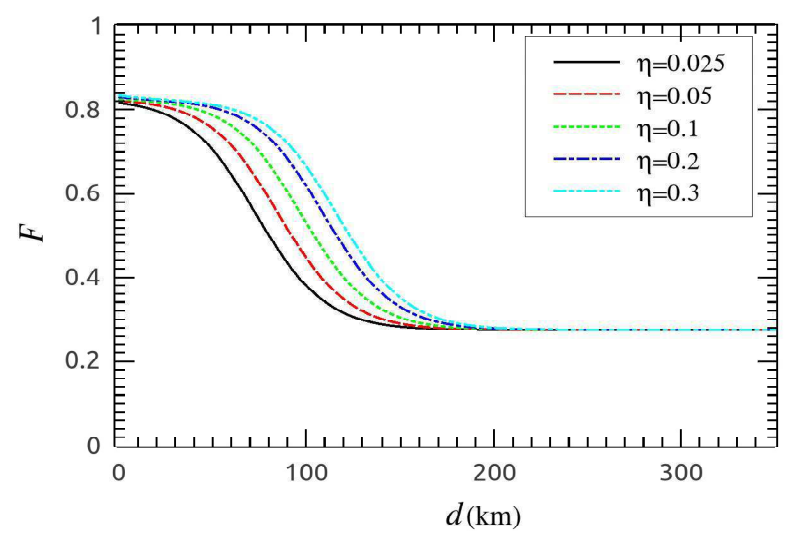

FIG. 5. (Color online) Fidelity $F$ is plotted vs distance $d \mathrm{~km}$ for $\chi=0.316$ and dark-count probability, $\zeta_{\mathrm{dc}}=10^{-5}$, and $\eta=0.025$ to 0.3 (from lower to higher fidelity curves).

We now apply our model to a recent experiment in the next section.

\section{COMPARISON WITH EXPERIMENTAL QT}

In order to test the validity of our model of QT, we apply it to a recent experiment [16]. In this experiment, long distance QT is achieved for over $100 \mathrm{~km}$. We compare the fidelity of the teleported state with that of the experiment using the same values of resource parameters such as photon pair production rate, $\chi$, detectors efficiency, $\eta$, and dark count rate as in the experiment. This allows us to check the validity of our model for long dis- tance QT and to analyze the effect of transmission losses as well. It is important to note that in our theory transmission loss is included in the detection efficiency.

We compare the predictions of our model with a recent experiment of QT using polarization property of photons [16]. The conditions of this experiment are given by approximate values: detectors efficiency is $\eta \approx 0.236$, transmission line has loss coefficient $45 \mathrm{~dB}, \chi \approx \sqrt{0.1} \approx$ 0.316 and dark counts rate is $200 \mathrm{~s}^{-1}$. With gate time of the detector to be around 100ps, the dark count probabilities per gate would be $\zeta_{\mathrm{dc}}=2 \times 10^{-8}$. Figure. 6 demonstrates, our numerical simulation result of fidelity using these parameters. In our case the average fidelity is $80.4 \%$, whereas the average fidelity obtained in the experiment is $81.35 \%$ with \pm 1 standard deviation for $|\mathrm{H}\rangle,|\mathrm{V}\rangle$ and $| \pm\rangle$ teleported states. It is interesting to

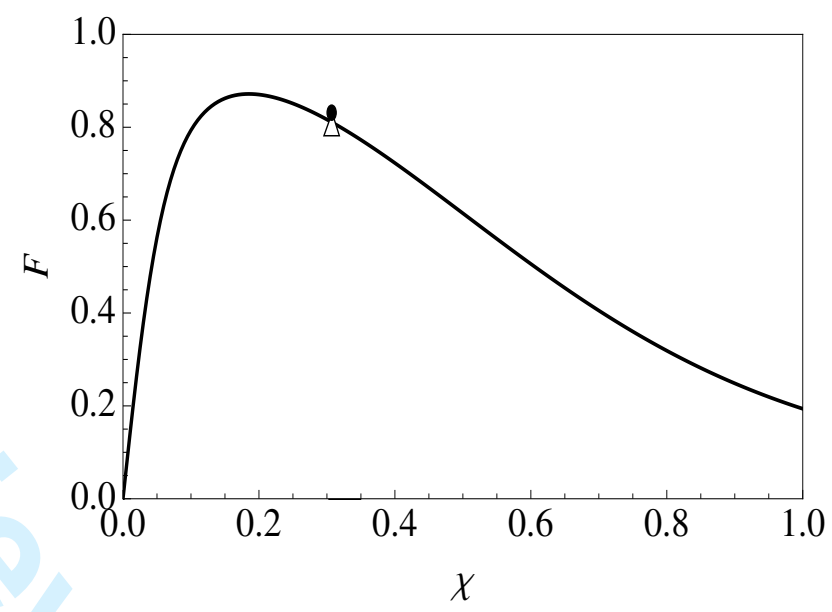

FIG. 6. Fidelity $F$ versus the square root of photon-pair production rate, $\chi$, with fixed dark-count rate $200 \mathrm{~s}^{-1}$ and detectors efficiency value: $\eta=0.7463 \times 10^{-5}$ including transmission losses. Calculated average fidelity is $80.4 \%$, at $\Delta$, and experimental value is $81.35 \%$, at $\bullet$.

observe that the photon-pair production rate of the PDC source used in this experiment, $\chi \approx 0.316$, lies far beyond its optimal value. The small difference in the calculated value by our model and the experimental value of average fidelities is due to the fact that detectors inefficiency and the multi-photon pair production are not the only practical limitation that effect the fidelity. Other limitations include the imperfect entanglement generated by the source, even in the scenario where only one single pair is created. In addition multimode analysis of the setup is needed for exact modeling of the experiment.

Figure 7 shows the variation of the fidelity with distance for the same experimental parameters. It is apparent that with these parameters, a maximum distance of around $100 \mathrm{~km}$ can be achieved. After this distance, the dark counts become effective and the fidelity decreases abruptly. The initial sub-unit fidelity is due to the presence of the multipairs, which lead to spurious coincidences. For detectors with unit efficiency and zero dark counts, visibility will saturate to this sub-unit fidelity for 
asymptotically large distance.

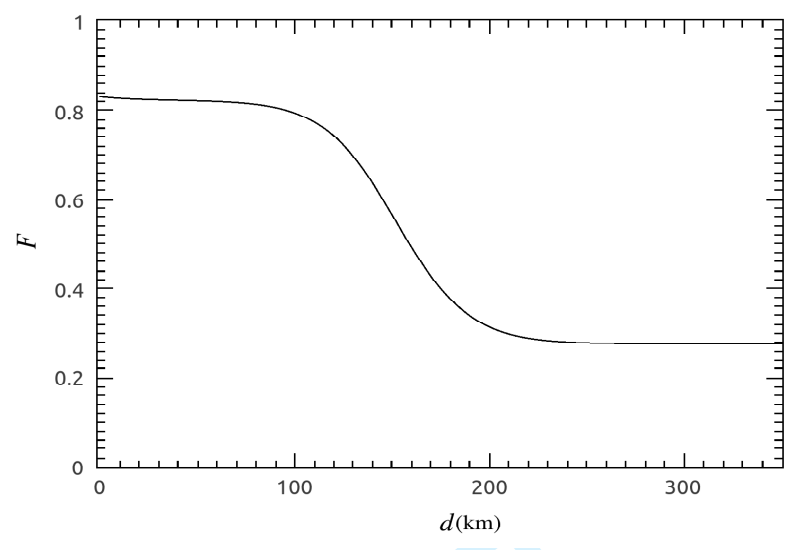

FIG. 7. Fidelity $F$ vs distance $d$ with dark-count rate $200 \mathrm{~s}^{-1}$ and detectors efficiency $\eta=0.236$ at $\chi=0.315$.

\section{CONCLUSION}

We have developed the model for practical quantum teleportation. We have used spontaneous parametric down conversion (SPDC) TYPE-II source for pre-shared entangled pair between the sender and receiver. Performing Bell measurement on the unknown state and the sender part of the entangled pair, we are able to find the teleported state. We have incorporated the faulty apparatus including multipair SPDC sources, detector inefficiencies and dark counts and channel losses. We have got a closed form for the teleported state. This teleported state has led us to calculate the fidelity of the teleported state with the desired unknown quantum state in terms of the nonlinearity, $\chi^{(2)}$, of the source, the detectors' efficiency $\eta$ and dark counts probability $\zeta_{\mathrm{dc}}$.

We show the variation of fidelity with the photon pair production rate, $\chi$, while fixing either dark counts, $\zeta_{\mathrm{dc}}$, and varying detectors efficiency, $\eta$, or vice a versa. Our theory gives expected results of the variation of the fidelity with the efficiency $\eta$ and dark count probability of detectors. By increasing the photon pair production rate $\chi$, the fidelity first increases for low $\chi$ and after reaching a maximum, it decreases as the multipair effect becomes dominant. This dependence is due to the fact that for small value of $\chi$ the probability of photon pair generation is small and thus the fidelity obtained is small because we do not have entangled photon pair due to vacuum component. As soon as $\chi$ reaches certain value which generates entangled photon pair with very less multi pair photons generation, the fidelity reaches certain maximum value which is far greater than the classical value 0.66 [18]. However, the high photon pair production rate is counterproductive and if we increase $\chi$ beyond a certain value, the fidelity decreases. We have also analyzed the dependence of communication distance on various parameters and found that detector inefficiency and dark counts limit the distance to few hundred kilometers. A very high fidelity can be attained for arbitrarily large distances if highly efficient detectors with very low dark count rate, such as superconducting nanowire detectors [19] are used. The sub unit fidelity in this case would be due to multipair effect of the source and the fidelity will saturate to this value for arbitrary distances.

We apply our model to a recent experiment on quantum teleportation [16]. Using same practical parameters given in [16], we obtained average fidelity of $80.4 \%$. Our calculated fidelity is in good agreement with the experimental value of $81.35 \%$. Thus our model fits very well to the real world experimental conditions. Our model can thus be used to predict the optimal parameters for the resources used to get the desired fidelity.

\section{ACKNOWLEDGMENTS}

We thank Barry C. Sanders for helpful comments and valuable discussions.
[1] C. H. Bennett, G. Brassard, C. Crépeau, R. Jozsa, A. Peres, and W. K. Wootters, Phys. Rev. Lett. 70, 1895 (1993), URL http://link.aps.org/doi/10.1103/ PhysRevLett.70.1895.

[2] D. Gottesman and I. L. Chuang, Nature 402, 390 (1999), ISSN 0028-0836, URL http://dx.doi.org/10. $1038 / 46503$.

[3] Y. Xia, J. Song, P.-M. Lu, and H.-S. Song, Journal of Applied Physics 109, 103111 (2011), http://aip.scitation.org/doi/pdf/10.1063/1.3592290, URL http://aip.scitation.org/doi/abs/10.1063/1. 3592290.

[4] M. Lu, Y. Xia, L.-T. Shen, J. Song, and N. B. An, Phys. Rev. A 89, 012326 (2014), URL http://link.aps.org/
doi/10.1103/PhysRevA.89.012326.

[5] D. Bouwmeester, J.-W. Pan, K. Mattle, M. Eibl, H. Weinfurter, and A. Zeilinger, Nature 390, 575 (1997).

[6] Zhao Zhi, Chen Yu-Ao, Zhang An-Ning, Yang Tao, Briegel Hans J., and Pan Jian-Wei, Nature 430, 54 (2004), ISSN 0028-0836.

[7] J.-W. Pan, D. Bouwmeester, H. Weinfurter, and A. Zeilinger, Phys. Rev. Lett. 80, 3891 (1998), URL http://link.aps.org/doi/10.1103/PhysRevLett. 80. 3891.

[8] Q. Zhang, A. Goebel, C. Wagenknecht, Y.-A. Chen, B. Zhao, T. Yang, A. Mair, J. Schmiedmayer, and J.W. Pan, Nat Phys 2, 678 (2006), URL http://dx.doi. org/10.1038/nphys417. 
[9] B. J. Metcalf, J. B. Spring, P. C. Humphreys, N. ThomasPeter, M. Barbieri, W. S. Kolthammer, X.-M. Jin, N. K. Langford, D. Kundys, J. C. Gates, et al., Nat Photon 8, 770 (2014), URL http://dx.doi.org/10.1038/ nphoton.2014.217.

[10] I. Marcikic, H. de Riedmatten, W. Tittel, H. Zbinden, and N. Gisin, Nature 421, 509 (2003), ISSN 0028-0836, $10.1038 /$ nature01376.

[11] R. Ursin, T. Jennewein, M. Aspelmeyer, R. Kaltenbaek, M. Lindenthal, P. Walther, and A. Zeilinger, Nature 430, 849 (2004), ISSN 0028-0836, 10.1038/430849a.

[12] X.-M. Jin, J.-G. Ren, B. Yang, Z.-H. Yi, F. Zhou, X.-F. Xu, S.-K. Wang, D. Yang, Y.-F. Hu, S. Jiang, et al., Nat Photon 4, 376 (2010), ISSN 1749-4885, 10.1038/nphoton.2010.87.

[13] P. Kok and S. L. Braunstein, Phys. Rev. A 61, 042304 (2000), URL http://link.aps.org/doi/10.
1103/PhysRevA.61.042304.

[14] M. Takeoka, R.-B. Jin, and M. Sasaki, New Journal of Physics 17, 043030 (2015), URL http://stacks.iop. org $/ 1367-2630 / 17 / i=4 / a=043030$.

[15] A. Scherer, R. B. Howard, B. C. Sanders, and W. Tittel, Phys. Rev. A 80, 062310 (2009), URL http://link.aps. org/doi/10.1103/PhysRevA.80.062310.

[16] J. Yin, J.-G. Ren, H. Lu, Y. Cao, H.-L. Yong, Y.-P. Wu, C. Liu, S.-K. Liao, F. Zhou, Y. Jiang, et al., Nature 488, 185 (2012).

[17] S. D. Bartlett, D. A. Rice, B. C. Sanders, J. Daboul, and H. de Guise, Phys. Rev. A 63, 042310 (2001), URL http: //link.aps.org/doi/10.1103/PhysRevA.63.042310.

[18] S. Popescu, Phys. Rev. Lett. 72, 797 (1994), URL http: //link.aps.org/doi/10.1103/PhysRevLett.72.797.

[19] F. Marsili, V. Verma, J. Stern, S. Harrington, A. Lita, T. Gerrits, I. Vayshenker, B. Baek, M. Shaw, R. Mirin, et al., Nature Photonics 7, 210 (2013). 